\title{
THE TREATMENT OF CONGENITAL VERTICAL TALUS
}

\author{
J. M. FITTON, A. B. NEVELÖS
}

From St James's Hospital, Leeds

\begin{abstract}
Operative correction of congenital vertical talus in nine feet in six patients is described. The operation consists of the comprehensive lengthening of the tendons in the foot and full peritalar release without excision of the navicular; it is performed through a dorsal transverse incision.
\end{abstract}

Congenital vertical talus is a rare deformity; its aetiology is uncertain and its treatment controversial. A large proportion of the children with this deformity have well-recognised neuromuscular abnormalities in the calf and foot the cause of which, meningomyelocele for instance, may be apparent, but some others with a congenital vertical talus have no demonstrable neuromuscular disturbance in the lower limbs. The pathological anatomy of congenital vertical talus has been studied by Patterson, Fitz and Smith (1968) and by Drennan and Sharrard (1971), and Colton (1973) and more recently Clark, D' Ambrosia and Ferguson (1977) have summarised the earlier literature on its treatment. The authors of these and other papers (Eyre-Brook 1967) advised excision of the navicular to reduce the length of the inner longitudinal arch of the foot and thus make repositioning of the talus possible. In our view, the apparent shortening of this arch is due to the backward and outward displacement of the calcaneus. Repositioning of the calcaneus restores the equality of the inner and outer arches of the foot, making it possible to retain the navicular, with which at least part of the movement of inversion can be regained. In this paper we describe the operative procedure used for the correction of congenital vertical talus in nine feet in six patients.

\section{THE OPERATION}

The principle of the operation is a full open peritalar release, including the calcaneocuboid joint, and the lengthening of all the dorsal and peroneal tendons in the foot and the tendo calcaneus. In the first two patients this operation was performed in two stages, through two separate incisions (posteromedial and lateral). In the last five years, however, we have found that complete and lasting correction was possible in one stage, using a dorsal transverse incision and a small posterior incision for the lengthening of the tendo calcaneus. The transverse incision starts from the front of the medial malleolus and ends just antero-inferiorly to the lateral malleolus. Through this incision the tendons of the tibialis anterior, the extensors and the peronei can be lengthened. The neurovascular bundle is carefully preserved and a full peritalar release is carried out, including complete division of the capsule of the calcaneocuboid joint. The talus can now be reduced with relative ease. It is fixed in its reduced position with a Kirschner wire passed through the dorsum of the foot. The tendons are then repaired and the skin is closed. The foot is held in a plaster of Paris cast for six weeks altogether. The cast is changed at three weeks, when the Kirschner wire can be removed.

\section{THE PATIENTS}

Although four of the six patients in this series had closed congenital abnormalities of the lumbosacral spine, none had any demonstrable neurological abnormality in the lower limbs. The calf of the affected leg appeared wasted, particularly when in the unilateral cases comparison was made with the unaffected leg, but the typical "pipe-stem" calf encountered in severe club foot was not seen. This relative muscle wasting remained noticeable after operation.

\section{CASE HISTORIES}

Case 1. This girl was born with a spina bifida occulta from $L 3$ to $S 1$ and a right-sided vertical talus. She was one month old when first examined. Correction by splinting failed. At the age of four months operative reduction was carried out. Through a lateral incision the tendons of the peronei, the tibialis anterior and the extensors were lengthened, and a peritalar release was carried out. Reduction was possible, and the foot was held in a cast for six weeks. At the age of eight months the shape of the foot was unsatisfactory and at this time, through a posteromedial incision, the tibialis anterior tendon and tendo calcaneus were again lengthened. A further peritalar release was carried out; the reduction was held with a Kirschner wire for three weeks, and the child was in a plaster of Paris cast for three months. Eight years after operation the shape of the foot was satisfactory; the child led a normal, active life, wearing normal shoes. The range of movements was not recorded, and the child has not attended for follow-up.

Case 2. This girl was born with spinal bifida occulta at L3-4-5 and a left vertical talus (Figs 1 and 2). Corrective splintage was started on the second day. At the age of two months, through a posterolateral curved incision, the described procedure was carried out, and the talus was held in position with a Kirschner wire. The wire was removed at three weeks, but plaster fixation continued for six weeks. At the age of two

J. M. Fitton, M.B.E., F.R.C.S., Consultant Orthopaedic Surgeon A. B. Nevelös, F.R.C.S., Senior Registrar (Orthopaedics) Requests for reprints should be sent to Mr J. M. Fitton. 


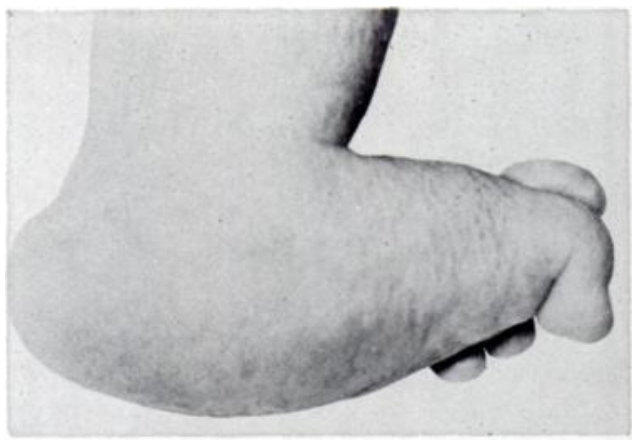

Fig. 1

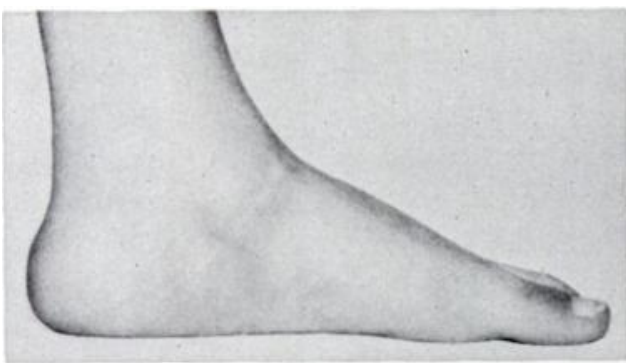

Fig. 3

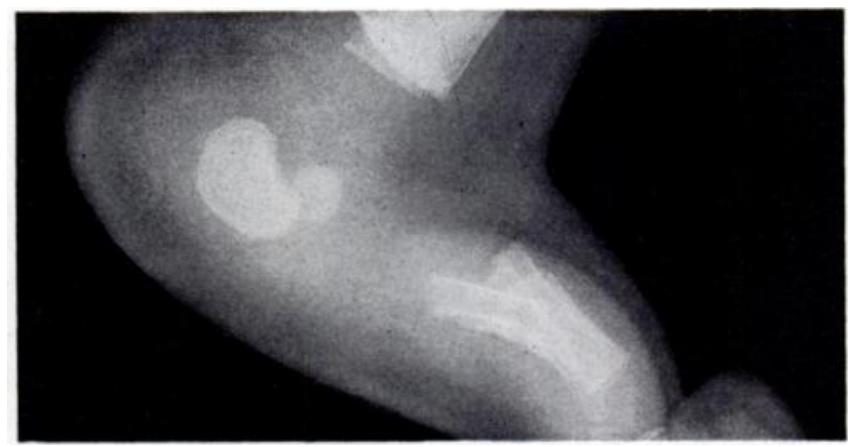

Fig. 2

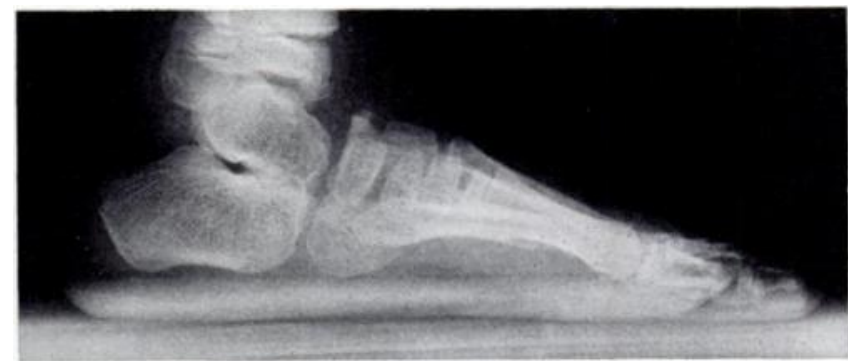

Fig. 4

Case 2. Figures 1 and 2-Clinical photograph and radiograph of the left foot of a girl, aged two weeks, with congenital vertical talus. Forced plantar flexion does not alter the position of the talus. Figures 3 and 4-Eight years after operation the shape of the foot is satisfactory and the talonavicular relationship is near normal.

years the position had deteriorated, and so at this time, through a medial incision. the tibialis anterior tendon and the extensors were again lengthened. The talus was released in the midtarsal joint and repositioned. A Kirschner wire held the reduction for three weeks, and the total time in plaster of Paris was six weeks. Radiographs and photographs taken eight years after operation are shown in Figures 3 and 4 .

Case 3. This girl was born with extensive closed lumbar spinal anomalies and bilateral vertical talus. After initial splintage, at the age of three months both feet were corrected using the transverse incision; the tendini calcanei were lengthened through separate posterior incisions. After three weeks the wires used for fixation were removed and the feet were supported in firm bandages. When the child was seen five years after operation she had 10 degrees of dorsiflexion and 10 degrees of plantar flexion from the neutral position of the ankles; inversion and eversion of the heel and the forefoot was 1 to 2 degrees only. She was able to lead an active life and wore normal shoes.

Case 4. This West Indian girl was born with bilateral vertical talus. No spinal abnormalities were noted. Figure 5 shows the clinical appearance of her feet and Figure 6 the radiograph of the right foot when she was first seen at the age of two months. At the age of six months both feet were operated upon. The reduction was held with a Kirschner wire for three weeks. Four months after this operation it became evident that the tibialis anterior tendon on the right foot was not acting; this foot was re-explored and it was found that the suture had failed. The tendon was dissected free and inserted into the navicular, and the foot was held in a cast for four months. Figures 7 to 10 show the photographs and radiographs of this child's feet taken four years after operation. She had 30 degrees of dorsiflexion and 20 degrees of plantar flexion in both ankles from neutral; she had no

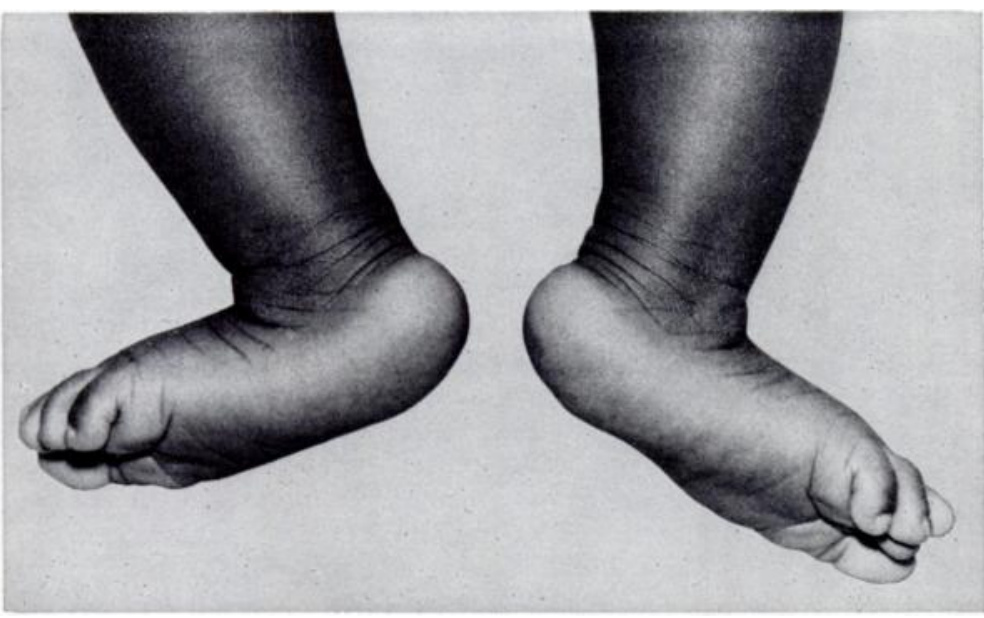

Fig. 5

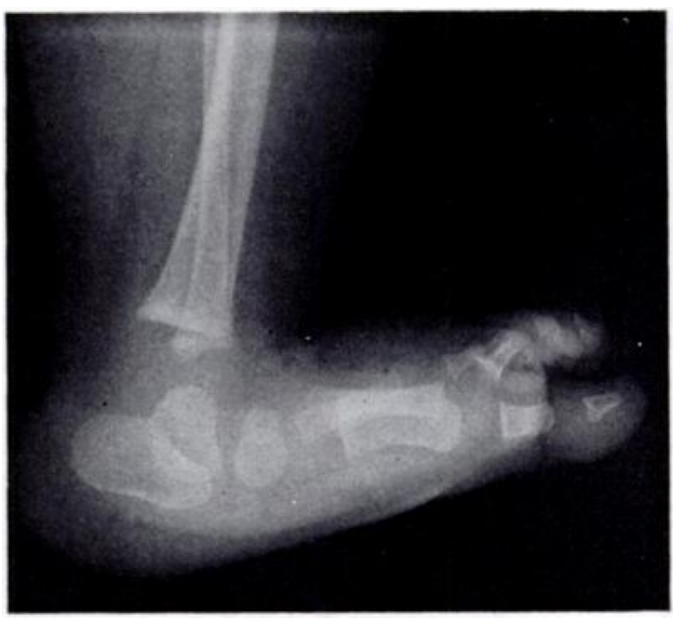

Fig. 6

Case 4. Figure 5-Clinical photograph of the feet of a girl, aged two months, with bilateral congenital vertical talus. Figure 6-Radiograph of the right foot of the same patient. 


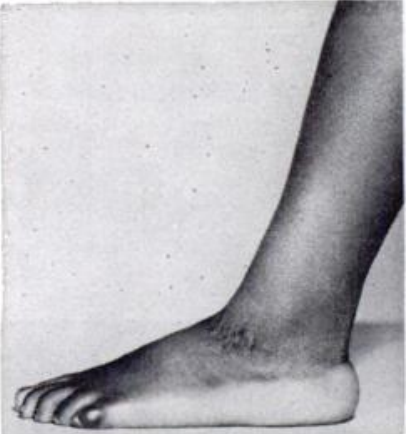

Fig. 7
Fig. 8
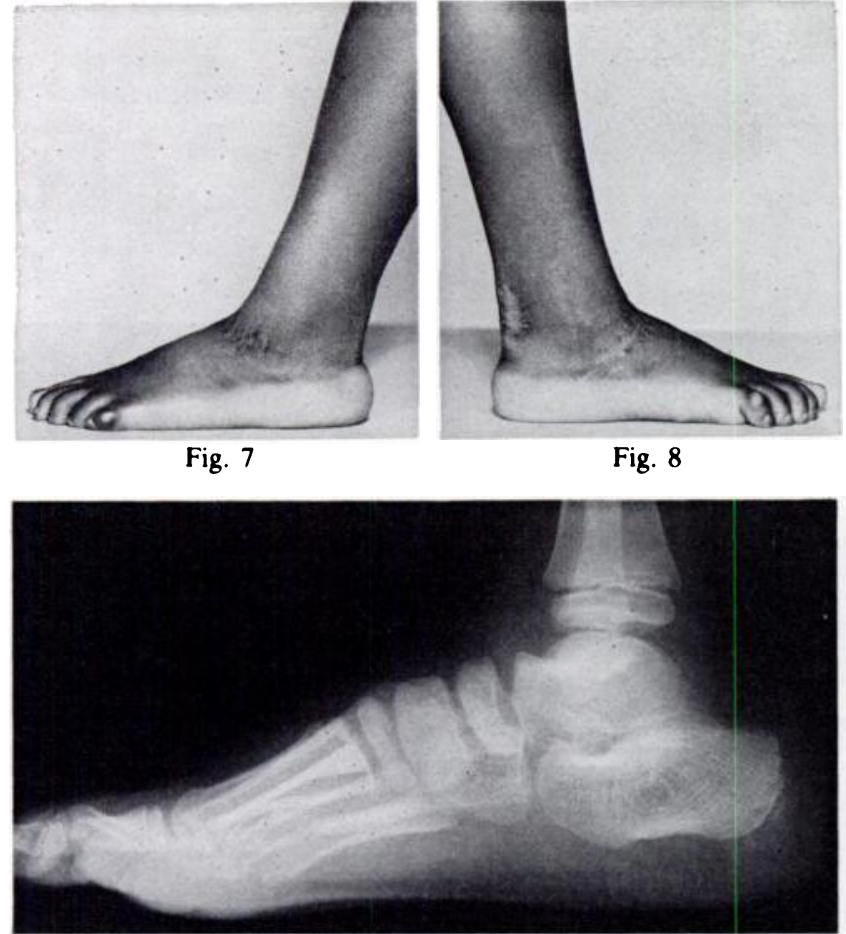

Fig. 9

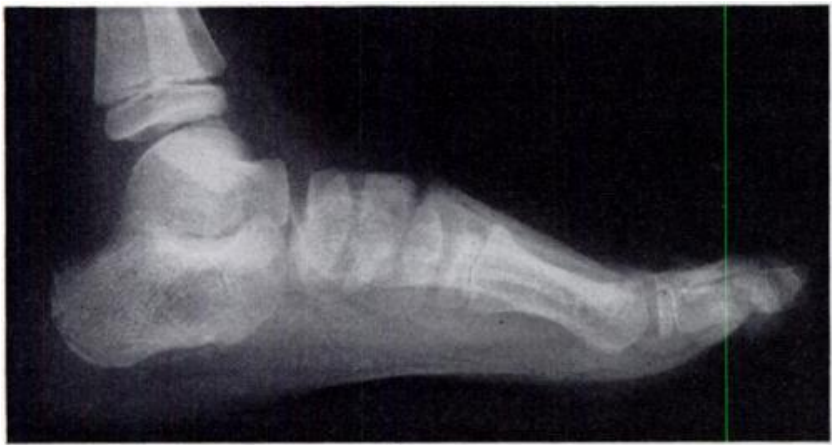

Fig. 10

Case 4. Clinical photographs and radiographs four years after operation. The scars are visible, and the shape of the feet is satisfactory.

inversion or eversion of the heel. She had 10 degrees of inversion and 10 degrees of eversion of the right forefoot, and 20 degrees of inversion and 10 degrees of eversion of the left forefoot.

Case 5. This girl was born with bilateral vertical talus. No spinal abnormalities were noted. Closed reduction was attempted first in plaster casts. then at the age of two months the feet were manipulated under general anaesthesia with radiographic control. The correction obtained. however, was unsatisfactory, thus at the age of six months both feet were operated upon. After reduction, the talus was held with a Kirschner wire for three weeks. The casts were changed at three weeks and removed at two months. The shape of the feet was satisfactory a year and a half after operation. The patient had 30 degrees of dorsiflexion and 30 degrees of plantar flexion of the right ankle, and 20 degrees of dorsiflexion and 25 degrees of plantar flexion of the left ankle; she had no inversion or eversion of the heel and no inversion of the forefeet. but had 15 degrees of eversion of both forefeet.

Case 6. This boy was born with bilateral hydroceles, a hemivertebra at S2 level and a left vertical talus. He was referred for orthopaedic advice at the age of eight months. and had an operative correction of the vertical talus two months later. The described operation was performed and three months later the child was able to walk. He had 10 degrees of dorsiflexion and 5 degrees of plantar flexion in the affected ankle. from the neutral position; he had only a minimal amount of inversion and eversion of the heel. and the forefoot everted to 10 degrees and inverted to 5 degrees.

\section{DISCUSSION}

Study of the patients in this small series reveals important factors regarding the treatment of congenital vertical talus. It is evident that whatever the aetiology of the deformity, conservative or manipulative treatment is unsuccessful. We would now recommend operative treatment at the age of about three to six months, when the operation described above becomes feasible. We believe that the sooner this deformity is corrected fully, the less remodelling of the talus is needed. Patterson $e t$ al. (1968) showed that in this condition the talus is already deformed at birth, and early full reduction may facilitate remodelling.

We believe that excision of the navicular is not necessary in the correction of congenital vertical talus. The complete division of the calcaneocuboid joint and the comprehensive lengthening of tendons are the key elements of the operation described. The anatomical studies published (Patterson et al. 1968; Drennan and Sharrard 1971) clearly indicate the importance of such a radical approach. The transverse incision is convenient, it heals well and leaves an acceptable non-adherent scar. Photographs and radiographs at long-term follow-up one to eight years later show the satisfactory shape of the foot and the near normal alignment of the tarsal bones after this operation.

Although the calf of the affected leg will remain abnormal in appearance even after operative repair, this abnormality is not striking. The children are able to wear normal shoes and to lead an active life. The range of movement remains restricted and seems to decrease in the years after the operation, but this restriction appears to have little effect on the functional ability of the children.

\section{REFERENCES}

Clark, M. W., D'Ambrosia, R. D., and Ferguson, A. B., Jun. (1977) Congenital vertical talus. Journal of Bone and Joint Surgery. 59-A, 816-824.

Colton, C. L. (1973) The surgical management of congenital vertical talus. Journal of Bone and Joint Surgery, 55-B, 566-574.

Drennan, J. C., and Sharrard, W. J. W. (1971) The pathological anatomy of convex pes valgus. Journal of Bone and Joint Surgery: 53-B, $455-461$

Eyre-Brook, A. L. (1967) Congenital verical talus. Journal of Bone and Joint Surgery, 49-B, 618-627

Patterson, W. R., Fitz, D. A., and Smith, W. S. (1968) The pathologic anatomy of congenital convex pes valgus. Journal of Bone and Joint Surgery, 50-A, 458-466 